\title{
Investigations of Optical Coulomb Blockade Oscillations in Plasmonic Nanoparticle Dimers
}

\author{
Lamessa Gudata, ${ }^{1}$ Jule Leta Tesfaye, ${ }^{1,2}$ Abela Saka, ${ }^{1}$ R. Shanmugam, ${ }^{3}$ \\ L. Priyanka Dwarampudi, ${ }^{4}$ Nagaraj Nagaprasad $\left(\mathbb{D},{ }^{5}\right.$ B. Stalin $\mathbb{D},{ }^{6}$ \\ and Ramaswamy Krishnaraj $\mathbb{1}^{2,7}$ \\ ${ }^{1}$ Department of Physics, College of Natural and Computational Science, Dambi Dollo University, Ethiopia \\ ${ }^{2}$ Centre for Excellence-Indigenous Knowledge, Innovative Technology Transfer and Entrepreneurship, \\ Dambi Dollo University, Ethiopia \\ ${ }^{3}$ TIFAC, CORE-HD, JSS College of Pharmacy, JSS Academy of Higher Education \& Research, Ooty, Nilgiris, Tamil Nadu, India \\ ${ }^{4}$ Department of Pharmacognosy, JSS College of Pharmacy, JSS Academy of Higher Education \& Research, Ooty, Nilgiris, \\ Tamil Nadu, India \\ ${ }^{5}$ Department of Mechanical Engineering, ULTRA College of Engineering and Technology, Madurai, 625104 Tamil Nadu, India \\ ${ }^{6}$ Department of Mechanical Engineering, Anna University, Regional Campus Madurai, Madurai, 625019 Tamil Nadu, India \\ ${ }^{7}$ Department of Mechanical Engineering, College of Engineering and Technology, Dambi Dollo University, Ethiopia
}

Correspondence should be addressed to Ramaswamy Krishnaraj; prof.dr.krishnaraj@dadu.edu.et

Received 11 November 2021; Accepted 21 December 2021; Published 15 January 2022

Academic Editor: Bharath Govindan

Copyright (c) 2022 Lamessa Gudata et al. This is an open access article distributed under the Creative Commons Attribution License, which permits unrestricted use, distribution, and reproduction in any medium, provided the original work is properly cited.

\begin{abstract}
The exploration of Coulomb blockade oscillations in plasmonic nanoparticle dimers is the subject of this study. When two metal nanoparticles are brought together at the end of their journey, tunnelling current prevents an infinite connection dipolar plasmon and an infinite amplification in the electric fields throughout the hot spot in between nanoparticles from occurring. One way to think about single-electron tunnelling through some kind of quantum dot is to think about Coulomb blockage oscillations in conductance. The electron transport between the dot and source is considered. The model of study is the linear conductance skilled at describing the basic physics of electronic states in the quantum dot. The linear conductance through the dot is defined as $G=\lim _{\longrightarrow 0}(I / V)$ in the limit of infinity of small bias voltage. We discuss the classical and quantum metallic Coulomb blockade oscillations. Numerically, the linear conductance was plotted as a function gate voltage. The Coulomb blockade oscillation occurs as gate voltage varies. In the valleys, the conductance falls exponentially as a function gate voltage. As a result of our study, the conductance is constant at high temperature and does not show oscillation in both positive and negative gate voltages. At low temperature, conductance shows oscillation in both positive and negative gate voltages.
\end{abstract}

\section{Introduction}

Classical electromagnetism forecasts an infinite of the redshift hybridized noble metal nanoparticle plasmon polariton. The surface-to-surface distance approaches zero when there is a rapid increase in the field strength between the hotspot of two metallic nanopatrticles. One theory holds that quantum mechanical tunnelling of electrons prevents either type of deviations [1]. Additionally, the tunnelling current mini- mizes the accumulation of charge opposing surfaces. As a result, decreases in the electric field strength within the hotspot result in a seamless transition from either the dipolar bonding plasmon to the charge transfer plasmon. A high degree of agreement exists between quantum mechanical theories and experimental results [2]. Quantum tunnelling accounts for the vast majority of the optical response of couples of plasmonic nanoparticles along within close proximity [3]. Recently, tunnelling of electrons in quantum plasmonics 
was thought to be a continuous phenomenon, when the conductivity across two nanoparticles increased progressively in decreasing range [4]. The development of charge transfer should be prevented by the Coulomb blockage of tunnelling current. The device should remain in the classical electromagnetism regime since a particular quantity of potential energy is achieved in the gap across plasmonic nanoparticles. With the emergence of supercomputers, numerical computations of electron transport across quantum dots have become increasingly essential in the field of physics. A computational approach allows for the derivation of the electronic characteristics and composition of a system of particles from the fundamental elements of the system and their interactions with one another. The quantum dot [5] is a system of particles that is presently receiving much attention. An artificial system made up of multiple interacting electrons that are constrained to limited regions among layers of metals is known as a quantum well.

In metal, a finite number of electrons are constrained. The possibility of evaluating and tuning features linked to quantum mechanical processes and charge quantization, as well as the ease with which such small devices may be constructed, encourages the interest in their fabrication. A very small capacitance is produced by a dot with a size that is in the region of the Fermi wavelength. Consequently, the electrons occupy separate quantum levels in dots, and the level spacing $E$ is the spacing across neighbouring levels in a dot grid system. In a way to extend an electron to the dot or take an electron from the dot, a specific quantity of energy $E_{c}$ is required, which varies based on the charging energy $E_{c}$. Atomic properties [6] are strikingly comparable to these features. As a result, quantum dots are considered "manufactured atoms" in some circles. The features of quantum dots, such as their spectrum, present a difficult advantage. It is possible to attach source and drain contacts to samples that are adjustable by an outside gate and that have source and drain contacts. As a result, it is possible to measure the transit through the dot. The tenability of variables has a significant impact on the amount of work that can be done in electron transport. With the addition of contacts to the dot, tunnelling of electrons away from and toward the dots becomes conceivable [7]. These result in a finite lifespan of the electron in the dot, producing a level broadening that is lower than the level spacing in the devices that we are interested in. A quantum dot is connected to three terminals by a quantum coupling. When the source and drain are in contact, particle exchange might occur. The third terminal, which is just electrically linked, is utilized as a gate electrode, shifting the energy spectrum of the dot as it passes through it. It is a quantum dot that has been attached to leads and the get capacitance that serves as the prototype for a solitary electron transistor. If we consider a realm in which temperature is substantially smaller than charging but still significantly greater than the level widening, the transport mechanism is controlled by a singular electron process. As with a transistor, the quantum dot operates similarly to a transistor in that the current flowing through it may be switched on and off by adjusting the gate voltage connected to it [8].

Quantum dots research embraces a variety of topics in physics, electrical and electronic engineering, chemistry, material science, biology, and medicine, where according to the demand of specific applications, different types of quantum dot structures are employed. Due to the fact that quantum dots could interconvert light and electricity in a controlled manner that is based on the size of the crystal, they can be used to inject directly charged particles [9]. One further use of quantum dots, and among the most intriguing interfaces of nanotechnology throughout the usage of a colloidal quantum dot in terms of speed and excitement, is in the field of biology [10]. There is a range of biological investigations in which typical fluorescent labels made from organic compounds fail to provide longterm sustainability or the ability to detect numerous signals at the same time. Their distinctive optical features made them appealing as fluorophores [11]. Applications of quantum in the area of biology are to investigate various cellular labelling mechanisms and develop drugs [12]. Generally, the applications of the quantum dots are diverse, and all could not be listed here in detail [13]. The confined electrons are held in place by a quantum dot, which would be a tiny area of a metal into which they might enter and exist due to tunnel contact. Charge transport through the quantum dots has been receiving significant theoretical and experimental interest. Research occurs on electron transport through the quantum dots in the noninteracting investigation to realize electron transport through the quantum dots at high temperature. On the other hand, there should be enough study for electrons to transport low temperature. The researcher is expected to fill this gap. The objective of the study is to investigate Coulomb blockade oscillations, conductance, and charge transport through the quantum dots and plot the normalized conductance as the function of gate voltage at nanoparticle plasmon dimers.

\section{Materials and Methodology}

The Coulomb barrier becomes significantly more visible as metallic nanoparticles within the sub-5 nm region become more readily available for manipulation. Scanning tunnelling microscopy [14] and electrochemistry with gold nanoparticles in the electrolyte [15] have both been used to observe Coulomb blockage occurring at ambient temperature. The quantum dot is perceived to be weakly coupled to two-electron reservoirs and to have a single electron energy level at $E_{p}(p$ $=1,2,3, \cdots)$; this is evaluated by handling the electronelectron interconnection in a mean-field (Hartee) approximation, which is a mean-field approximation of the electronelectron interaction [16]. In this chart, the stages are designated in ascending order and are evaluated in relation to the potential well's bottom. The location of the levels may be affected by the number of electrons in the molecule.

$$
\begin{aligned}
& f_{\mathrm{l}}\left(E-E_{\mathrm{F}}\right)=\frac{1}{1+e^{\left(E-E_{\mathrm{F}}\right) / K_{\mathrm{B}} T_{\mathrm{l}}}}, \\
& f_{\mathrm{r}}\left(E-E_{\mathrm{F}}\right)=\frac{1}{1+e^{\left(E-E_{\mathrm{F}}\right) / K_{\mathrm{B}} T_{\mathrm{r}}}},
\end{aligned}
$$


where $E_{\mathrm{F}}$ is the Fermi energy, $f$ is the Fermi-Dirac distribution, $K_{\mathrm{B}}$ is the Boltzmann constant, and $T_{1}$ and $T_{\mathrm{r}}$ are the temperature of left and right reservoirs, respectively. Through applying a conceivable variation $V$ between the left and right sides of the dot, a current $I$ could be transmitted through it. It is indicated by $\Gamma_{p}^{\mathrm{l}}$ and $\Gamma_{p}^{\mathrm{r}}$, respectively, that the tunnelling rate between level $P$ to the left and right reservoirs is greater than zero. In the presence of a potential $U$ imposed between both the source and drain reservoirs, the current will flow via the dot. The distribution of electrons in the quantum dot is altered as a result of this current. The stability distribution of electrons from between energy levels differs from the equilibrium distribution of electrons in the previous energy levels. The applied potential difference can now be considered a perturbation that can change the equilibrium distribution [17]. At $T=0$, the position of the conductance peaks as a function of gate voltage can be evaluated from a consideration of the equilibrium characteristics of the system only [18]. In linear response theory, the response is a linear function of perturbation [19].

$$
P\left(\left\{n_{i}\right\}\right)=P_{\text {eq }}\left(\left\{n_{i}\right\}\right)\left[1+\frac{e V}{K T} \psi\left(\left\{n_{i}\right\}\right)\right] .
$$

$\psi\left(\left\{n_{i}\right\}\right)$ is the correction term due to the application of perturbation. The equilibrium distribution is shifted by the factor of $\psi\left(\left\{n_{i}\right\}\right)$. The nonequilibrium probability evolves in time according to the master equation [20]. The master equation has gain terms and loses terms [21]. The (two-terminal) linear response conductance $G$ of the quantum dot is characterized by the expression $G=I / V$ in the limit $V \longrightarrow 0$. The stationary current through the left equals the right barrier and is provided by

$I=-e \sum_{p=1}^{\infty} \sum_{\left\{n_{i}\right\}} \Gamma_{p}^{1} P\left(\left\{n_{i}\right\}\right)\left\{\sigma_{n p, 0} f\left(E^{i, l}(N)-E_{\mathrm{F}}\right)-\sigma_{n p, 1\left[1-f\left(E^{f l}(N)-E_{\mathrm{F}}\right)\right]}\right\}$.

The first term expresses the chance of finding an electron with energy $E$ upon on the left side of the graph and an emptiness state on the right side of the graph [22]. The second summation is based on the total number of occupations that have been realized $\left\{n_{1}, n_{2, \ldots}\right\}=\left\{n_{i}\right\}$ from the energy levels in the quantum dot each with stationary probability $P_{\left\{\left\{n_{i}\right\}\right\}}$. The conductance through the quantum dot is for a given $N$ :

$G=\frac{e^{2}}{K T} \sum_{P=1}^{\infty} \sum_{N=1}^{\infty} \frac{\Gamma_{p}^{1} \Gamma_{p}^{\mathrm{r}}}{\Gamma_{p}^{1}+\Gamma_{p}^{\mathrm{r}}} P_{\mathrm{eq}}(N) F_{\mathrm{eq}}\left(E_{p \mid N}\right)\left[1-f\left(E_{p}+U(N)-U(N-1)-E_{\mathrm{F}}\right)\right]$.

Specifically, this distribution function describes the tunnelling of an electron with an initial condition $P$ in the dot [23]. When we are in the extreme temperature limit, we take the quantum into consideration and $K_{\mathrm{B}} T \gg \Delta E$. The extreme temperature range intersect with lower temperature range [24].
The conductance of the particular barriers and quantum dots at higher temperatures is irrelevant, as the discreteness of neither the stages nor the charging energy is essential. The conductance does never fluctuate in response to the gate voltage [25]. For low-temperature characteristics, $K T \ll \Delta E$ . All energy levels $E_{p}$ are small that $G$ is the highest if $1-f$ is maximum. The charging energy $U(N)$ of the dot difference is discretely due to the variation in number $N$ of electrons on the dot through tunnelling to or from the source leads, or it repeatedly varies due to the difference in the voltage on the external gate electrode [26]. In the lowtemperature region, $K T \ll \Delta E$; the term with $P=N=N_{\text {min }}$ gives the dominant contribution to the sum over $P$ and $N$ in the conductance. Then, the equation of normalized conductance in the low-temperature boundary limit is given by

$$
\frac{G}{G_{o}}=\frac{\sum_{N=1}^{\infty} e^{\left(N-N_{\min }\right)^{2} / 2 \sigma^{2}} \sum_{P=1}^{\infty} 1 / 1+e^{-\beta \Delta}}{\sum_{N=1}^{\infty} e^{\left(N-N_{\min }\right)^{2} / 2 \sigma^{2}}},
$$

where $\Delta=N E_{c}+P \Delta E-\left(E_{\mathrm{F}}+e V_{g}+E_{c}\right)$ and $G_{0}=e^{2} \Gamma_{p}^{\mathrm{r}}$ $\Gamma_{p}^{\mathrm{l}} / K T\left(\Gamma_{p}^{\mathrm{r}}+\Gamma_{p}^{\mathrm{l}}\right)$. As temperature decreases, the width of Gaussian distribution $\sigma_{N}$ decreases, and thus, $P(N)$ becomes sharply peaked around $N=N_{\min }$.

\section{Result and Discussion}

The Coulomb blockade with resonant tunnelling and the optical field driving the plasmon leads to the equilibration of the Fermi Level of plasmonic nanoparticles and molecular levels of intertion [27]. The Coulomb blockade oscillations break down when thermal energy is equal to charging energy. The numerical computation of electron transport through the quantum dot is performed [28]. The graphs of the results are discussed using the equations that we use in the method. Conductance cannot be evaluated analytically but can be evaluated numerically [29]. The usefulness of numerical computation is more recognized, and today, it is used in many domains of research and development. The normalized conductance is shown as a measure of gate voltage on a graph.

Numerically equation (5) is solved for different values of thermal energy and plotted normalized conductance also as a variable of gate voltage [30]. The plot of equation (5) is shown in Figure 1. For negative gate voltage conductance shows no oscillations but rather has a constant positive value of 274.62. For ETH $=1.46 \times 10^{-2}$ and negative gate voltage $V_{\mathrm{g}}=-0.037 \mathrm{~V}$, the conductance falls from $\max$ to $\mathrm{min}$ $\left(G_{\max }=274.62\right)$ to $\left(G_{\min }=12.05\right)$ and oscillate between $G_{\min }=12.05$ and $G_{\max }=71.31$. The constant value of conductance in the positive gate voltage is less than the constant value in the negative gate voltage. Oscillations occur in the positive gate voltage [31].

The plotted graph for low temperature in Figure 2 is shown. For both negative and positive gates, voltage conductance shows no oscillation. For negative voltage $V_{\mathrm{g}}=0.5 \mathrm{~V}$, the conductance falls $G_{\max }$ to $G_{\min }\left(G_{\max }=54.92\right.$ to $G_{\min }=$ 34.69) for $\mathrm{ETH}=1.82 E^{-2}$ and shows no oscillations [32]. As gate voltage increases, conductance decreases-approaching 


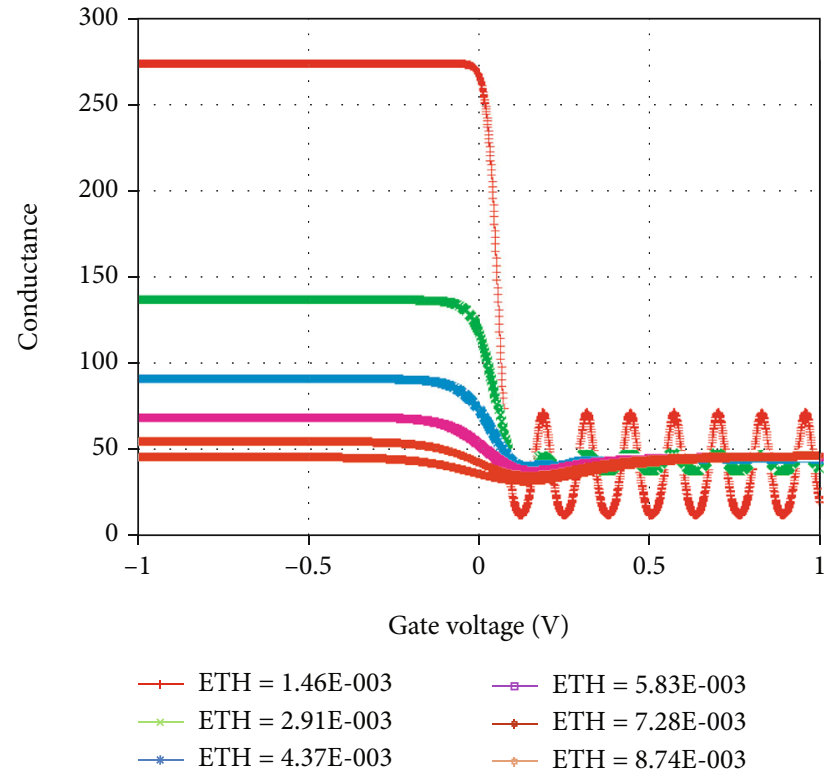

Figure 1: Normalized conductance versus gate voltage and plotted graph of equation (5).

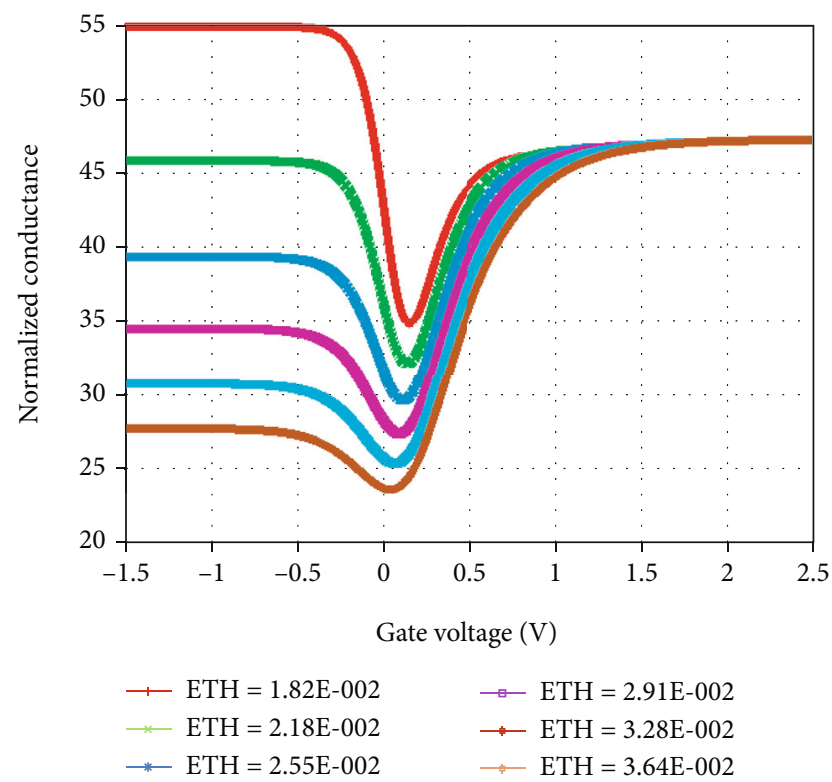

Figure 2: Blocking conditions. Normalized conductance versus gate voltage and a plotted graph of equation (5).

a minimum value in the positive gate voltage $\left(G_{\max }=34.69\right.$ )-and increases gradually becoming constant $(G=46.70)$. The conductance's blocking conditions ( $G$ is minimum) are fulfilled at different gate voltages [33]. As can be seen from the figure, at high temperature, the conductance does never exhibit oscillation as a variable of gate voltage. At a fixed gate voltage, the conductance valley (minima) through the dot is shifted to the right in energy proportional to the applied gate voltage. Transport blocked (Coulomb blockade) regions in

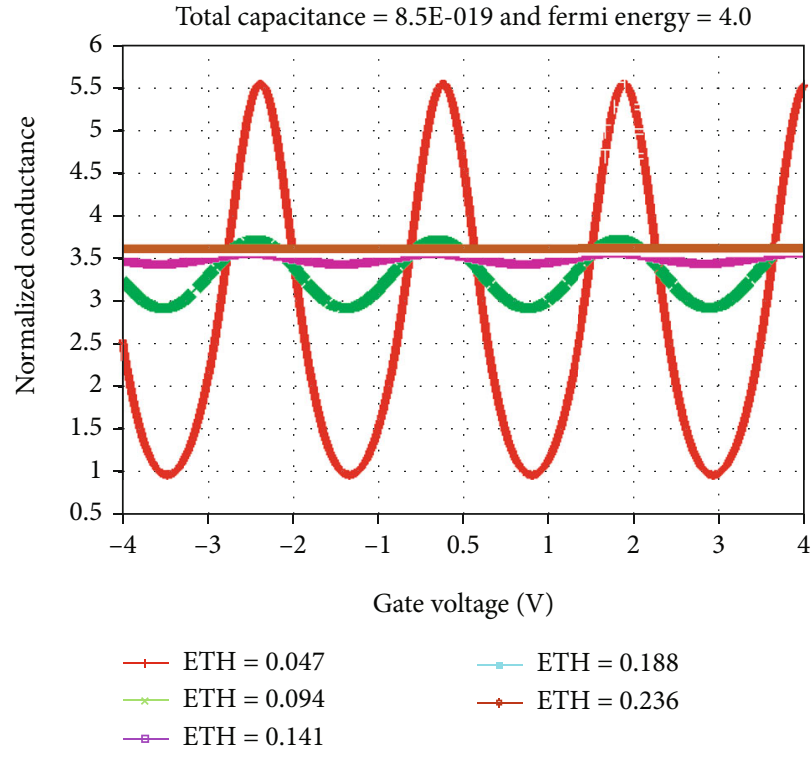

FIgURE 3: Normalized conductance versus gate voltage when $K_{\mathrm{B}} T$ $\ll \Delta E$ plotted using equation (5).

which no energy lies in the required energy window. No oscillations in both positive and negative gate voltages.

In Figure 3, we have plotted the normalized conductance as a variable of gate voltage for various capacitances with the same Fermi energy; the conductance becomes oscillatory for both negative and positive gate voltages [34]. For ETH $\approx$ 0.236 oscillation disappears, conductance becomes constant $G \approx 3.6$. Level spacing, capacitance, temperature, and chemical potential affect the oscillation nature of the conductance.

\section{Inference}

The close connections of our result and the objective of the study are in agreement with the theoretical explanation of the optical Coulomb blockade oscillations in plasmonic nanoparticle dimers. We briefly explained the appearances of the Coulomb blockade at high and low temperatures graphically.

\section{Conclusion}

Statistical computation of electron transport through the quantum dots is performed. The electron charge in the quantum dot is quantized. The conductance cannot be evaluated analytically but can be evaluated numerically. At high temperatures, the thermal energy is strong enough to overcome the repulsive of Coulomb force. The alternative pattern of peaks and suppressed region conductance as a variable of gate electrons are in the quantum dot. The normalized conductance is plotted as a variable of gate voltage for capacitance across the drain, capacitance across the gate voltage, capacitance across the source, charging energy, and the level spacing. The graphs of normalized conductance show a different behaviour, saturation, oscillation, oscillation depression, and varying amplitude of oscillation and period. 


\section{Data Availability}

The data used to support the findings of this study are included within the article.

\section{Disclosure}

This study was performed as a part of the employment of the authors.

\section{Conflicts of Interest}

The authors declare that there are no conflicts of interest.

\section{References}

[1] S. Stewart, Q. Wei, and Y. Sun, "Surface chemistry of quantum-sized metal nanoparticles under light illumination," Chemical Science, vol. 12, no. 4, pp. 1227-1239, 2021.

[2] J. J. Cully, J. L. Swett, K. Willick, J. Baugh, and J. A. Mol, "Graphene nanogaps for the directed assembly of singlenanoparticle devices," Nanoscale, vol. 13, no. 13, pp. 65136520, 2021.

[3] Y. Yusof, S. Moosavi, M. R. Johan et al., "Electromagnetic characterization of a multiwalled carbon nanotubes-silver nanoparticles-reinforced polyvinyl alcohol hybrid nanocomposite in X-band frequency," ACS Omega, vol. 6, no. 6, pp. 4184-4191, 2021.

[4] V. I. Kleshch, V. Porshyn, A. S. Orekhov, A. S. Orekhov, D. Lützenkirchen-Hecht, and A. N. Obraztsov, "Coulombblockade-controlled single-electron point source," 2020, https://arxiv.org/abs/2005.02358.

[5] D. Pan, H. Song, S. Zhang et al., "In situ epitaxy of pure phase ultra-thin InAs-Al nanowires for quantum devices," 2020, https://arxiv.org/abs/2011.13620.

[6] P. Strasberg, C. W. Wächtler, and G. Schaller, "Autonomous implementation of thermodynamic cycles at the nanoscale," Physical Review Letters, vol. 126, no. 18, p. 180605, 2021.

[7] T. Kanne, D. Olsteins, M. Marnauza et al., "Double nanowires for hybrid quantum devices," 2021, https://arxiv.org/abs/2103 .13938 .

[8] Y. Chen, S. Huang, J. Mu, D. Pan, J. Zhao, and H. Q. Xu, “A double quantum dot defined by top gates in a single crystalline InSb nanosheet," Chinese Physics B, vol. 30, no. 12, p. 128501, 2021.

[9] N. Kavokine, R. R. Netz, and L. Bocquet, "Fluids at the nanoscale: from continuum to subcontinuum transport," Annual Review of Fluid Mechanics, vol. 53, no. 1, pp. 377-410, 2021.

[10] M. S. Anwar and J. C. Fenton, "Gate-controlled conductance of superconducting $\mathrm{NbN}$ nanowires: coherent quantum phase-slips or coulomb blockade?," 2021, http://arxiv.org/ abs/2104.09331.

[11] C. J. Muller, "Decoupling environmental modes from tunneling electrons in a partially wet phase molecular mechanically contrallable break junction," 2021, http://arxiv.org/abs/2106 .01716 .

[12] M. Mirigliano and P. Milani, "Electrical conduction in nanogranular cluster-assembled metallic films," Advances in Physics: $X$, vol. 6, no. 1, p. 1908847, 2021.
[13] J. R. Bayogan, K. Park, Z. B. Siu et al., "Controllable p-n junctions in three-dimensional Dirac semimetal Cd3As2 nanowires," Nanotechnology, vol. 31, no. 20, p. 205001, 2020.

[14] I. S. Burmistrov, Y. Gefen, D. S. Shapiro, and A. Shnirman, "Mesoscopic stoner instability in open quantum dots: suppression of Coleman-Weinberg mechanism by electron tunneling," Physical Review Letters, vol. 124, no. 19, p. 196801, 2020.

[15] G. Scappucci, C. Kloeffel, F. A. Zwanenburg et al., "The germanium quantum information route," Nature Reviews Materials, vol. 45, pp. 1-18, 2020.

[16] F. Iachello and Y. Alhassid, "Nuclear Theory," Final Technical Report for DOE Grant DE-FG02-91ER40608 (No. DOEYALE-40608), Yale Univ, New Haven, CT (United States), 2020.

[17] Y. W. Lu, J. F. Liu, Z. Liao, and X. H. Wang, "Plasmonic-photonic cavity for high-efficiency single-photon blockade," Science China - Physics Mechanics \& Astronomy, vol. 64, no. 7, pp. 1-10, 2021.

[18] D. Sivun and T. A. Klar, "Optical coulomb blockade lifting in plasmonic nanoparticle dimers," Optics Express, vol. 28, no. 3, pp. 4115-4126, 2020.

[19] S. Kim, T. H. Le, Y. Choi et al., "Electrical monitoring of photoisomerization of block copolymers intercalated into graphene sheets," Nature Communications, vol. 11, no. 1, pp. 1-8, 2020.

[20] M. Mirigliano, S. Radice, A. Falqui, A. Casu, F. Cavaliere, and P. Milani, "Anomalous electrical conduction and negative temperature coefficient of resistance in nanostructured gold resistive switching films," Scientific Reports, vol. 10, no. 1, pp. 1-10, 2020.

[21] M. Tsutsui, S. Ryuzaki, K. Yokota et al., "Field effect control of translocation dynamics in surround-gate nanopores," Communications Materials, vol. 2, no. 1, pp. 1-9, 2021.

[22] A. Zalla, Quantum chemical simulations of the interaction of polyynes with silver nanoparticles, Europian Research Council, 2021.

[23] K. Willick, Carbon nanotube electromechanical systems: nonlinear dynamics and self-oscillation, [Ph.D. thesis], University of Waterloo, Ontario, Canada, 2020.

[24] S. P. Madhusudanan, M. S. Kumar, K. Mohanta, and S. K. Batabyal, "Photoactive $\mathrm{Cu}_{2} \mathrm{FeSnS}_{4}$ thin films: influence of stabilizers," Applied Surface Science, vol. 535, p. 147600, 2021.

[25] M. Napari, T. N. Huq, R. L. Hoye, and J. L. MacManus-Driscoll, "Nickel oxide thin films grown by chemical deposition techniques: potential and challenges in next-generation rigid and flexible device applications," InfoMat, vol. 3, no. 5, pp. 536-576, 2021.

[26] A. W. Elshaari, E. Büyüközer, I. E. Zadeh et al., "Strain-tunable quantum integrated photonics," Nano Letters, vol. 18, no. 12, pp. 7969-7976, 2018.

[27] X. Zhang, M. Q. Le, V. C. Nguyen et al., "Characterization of micro-ZnO/PDMS composite structured via dielectrophoresis-toward medical application," Materials \& Design, vol. 208, p. 109912, 2021.

[28] B. Wang, P. Nawarat, K. M. Lewis, P. Patsalas, and D. Gall, "Tunable infrared plasmonic properties of epitaxial TilxMgxN(001) layers," ACS Applied Materials \& Interfaces, vol. 13, no. 19, pp. 22738-22748, 2021.

[29] D. Sherman, J. S. Yodh, S. M. Albrecht, J. Nygård, P. Krogstrup, and C. M. Marcus, "Normal, superconducting 
and topological regimes of hybrid double quantum dots," Nature Nanotechnology, vol. 12, no. 3, pp. 212-217, 2017.

[30] A. Avila-García, A. Chaudhary, and H. Rojas-Chávez, "Iridium oxide films as propane sensors," Thin Solid Films, vol. 724, p. 138617, 2021.

[31] A. M. Hassan, F. Diab, E. F. Kotp, G. Al-Kashef, E. R. Shaaban, and A. E. Metawa, "Implantation of $\mathrm{Cu}$ onto $\mathrm{ZnTe}$ thin film using plasma focus device for optoelectronic applications," Optical Materials, vol. 117, p. 111182, 2021.

[32] S. Vaitiekènas, M. T. Deng, J. Nygård, P. Krogstrup, and C. M. Marcus, "Effective g factor of subgap states in hybrid nanowires," Physical Review Letters, vol. 121, no. 3, p. 037703, 2018.

[33] G. Badawy, S. Gazibegovic, F. Borsoi et al., "High mobility stemless InSb nanowires," Nano Letters, vol. 19, no. 6, pp. 3575-3582, 2019.

[34] R. L. O. Het Veld, D. Xu, V. Schaller et al., "In-plane selective area InSb-Al nanowire quantum networks," Communications on Physics, vol. 3, no. 1, pp. 1-7, 2020. 